\title{
Tips and tricks in femoropopliteal lesions
}

\author{
Hüsnü Değirmenci* and Eftal Murat Bakirci \\ Department of Cardiology, Faculty of Medicine, Erzincan Binali Yıldırım University, Turkey
}

Received: 18 January, 2021

Accepted: 02 March, 2021

Published: 03 March, 2021

*Corresponding author: Hüsnü Değirmenci, Department of Cardiology, Faculty of Medicine, Erzincan Binali Yıldırım University, Turkey, Tel: 05304645530 ;

E-mail: husnudr1982@gmail.com

Keywords: Femoropopliteal lesions; Morbidity; Mortality; Tips; Tricks

https://www.peertechzpublications.com

\section{Abstract}

Femoropopliteal lesions constitute an important part of lower extremity peripheral artery disease. Experience with these lesions contributes to reduction in morbidity and mortality. We have presented this article to bring tips and tricks to the literature that will contribute to morbidity and mortality reduction.

\section{Introduction}

Lower extremity peripheral artery disease is the progressive deterioration of lower extremity arterial blood circulation due to systemic atherosclerosis. Morbidity and mortality rates are high in this disease [1]. In the prevalence studies conducted in our country, the incidence in the society with increasing age is $20 \%-30 \%$ [2]. The diagnosis can be made by measuring the ankle brachial index. The incidence of coronary artery disease and congestive heart failure increases in individuals with lower extremity peripheral artery disease [3]. For this reason, we wrote this article on tips and tricks in Femoropopliteal Lesions (FPL), which have an important place in lower extremity peripheral artery disease.

\section{Femoropopliteal lesions}

Retrograde puncture and crossover (contralateral) technique: An $8 \mathrm{~F}$ sheat is entered by puncturing the contralateral femoral artery. Then, aortic bifurcation is reached with a 4 F LIMA catheter over a 0.035 inch hydrophilic wire. In the aortic bifurcation, the mouth of this catheter is turned towards the common iliac artery. Following this, the hydrophilic wire is advanced from the common iliac artery to the femoral artery. Later, the LIMA catheter is advanced over the hydrophilic wire to the other femoral artery. After this catheter is advanced into the femoral artery, the hydrophilic wire is removed. And the supracor or super amplatz stiff wire is advanced through the LIMA catheter in the femoral artery to wire the SFA. Later, the LIMA catheter is removed and sent over the supracor stiff wire, usually $90 \mathrm{~cm}$ sheatless, depending on the location of the lesion. Then, the supracor stiff wire is removed and the lesion is reached with a 0.014-0.018 inch compatible Minie support catheter, supercross microcatheter or other microcatheters with ASTATO 30 or TRESSURE 12 wires. ASTATO 30 is a penetration wire and is changed with TRESSURE 12 after the lesion is passed. The lesion is tried to be passed with various maneuvers. If there is a subintimal progression, it is tried to fall into the lumen by supporting the wire with a microcatheter. If the lumen cannot be reduced despite reaching the distal cap, the existing microcatheter can be changed with Bern, Quikcross or navicross microcatheter to direct the wire to the lumen. If the lesion cannot be passed with a 0.018 inch wire, a 0.035 wire can be used. If the 0.035 -inch wire lesion does not pass, the proximal cap can be crossed with the back stiff side of the wire in total occlusions. However, in this case, attention should be paid to perforation. As the proximal cap is passed, the microcatcher is advanced in the lesion and the procedure is continued with the soft part of the wire.

Although the guides recommend a stent in FPL, if there is no residual lesion after 4-5minutes of balloon inflation, or dissection limiting the flow, stenting is not recommended by experienced operators. After balloon dilatation, run off is evaluated in 3 stages. Stage 0 is the stage where there is no flow, stage 1 is the slow flow and stage 2 is the normal flow [4]. Stent restenosis is particularly high in long stents. If the residual lesion is below $50 \%$, grade $\mathrm{D}$ and higher dissection, ie dissection that limits the flow with peak translesional systolic pressure gradient above $10 \mathrm{mmHg}$, the procedure is considered successful. If there is more than $50 \%$ of the residual lesion and a dissection limiting the flow, a stent can be inserted [5]. Care 
should be taken not to lose the arteria femoralis profunda in FPL. If necessary, a filter can be placed in the ostialine for this artery. This artery is important, as it is an important source of collateral, nourishes the gluteal area, and the leg goes to amputation when occluded.

CT angiography has an important place in evaluating the length of the lesion and calcification in femoropopliteal lesions. Another point we should pay attention to in FPL is that balloons larger than $4 \mathrm{~mm}$ are not used for dilatation. This increases the risk of dissection. Instead, low diameter long balloons should be preferred. In FPL, Halberd or V18 can be used to go intraluminally in cases of total occlusion, while 0.018 inch wires such as Gladius or Gaiya can be used for subintimal or intraluminal access. Or, with ASTATO 30, it is tried to go intraluminally as far as possible, then pass the 0.035 -inch wire and push the subintimal wire quickly and fall into the lumen.

If the calcification determined in CT angiography is too much, atherectomy device can be used, but in this case, we recommend using a distal filter to avoid losing the distal run off (flow). Most long Superficial Femoral Artery (SFA) occlusions have collateral flow with different degrees of distal filling from the deep femoral artery, and such SFAs begin with the stump. For angiographic evaluation of the proximal stump, 35-40 degrees ipsilateral lateral angiography should be performed. If the SFA stump cannot be seen clearly, balloon dilatation can be performed by puncturing the distal SFA and removing the wire with a contralateral snare [6]

In case of flow-limiting dissection or suboptimal result, short balloon or self-expendable stent can be used. After recanalization with endoluminal or subintimal technique, balloon dilatation at 16 atm can be performed with balloons of 120-200 mm length in $>8 \mathrm{~cm}$ very long occlusions. However, attention should be paid to the balloon artery ratio to be $<1$, especially in focal lesions [7]. Otherwise, flow-limiting dissection may develop.

In case of short calcific proximal or ostial SFA lesion, failure of retrograde or antegrade intervention, need for intervention to common femoral artery in patients with bilateral iliac stent, complication of SFA closure device at proximal, need for embolization in case of iatrogenic injury in circumflex iliac or inferior epigastric artery, indication for middle SFA puncture [8].

Technical tips for overcoming SFA chronic total occlusion are as follows [9]:

- Using a rigid angled glidewire angled less than 5-6mm on the straight glide or Quick croos catheter support.

- Lumen re-entry devices (using angled microcatheters such as a bern or navicross catheter) when approaching the distal cap

- Using a stiff wire such as Cross-it $300-400$ for the $4 \mathrm{~F}$ bernstein microcatheter to perforate the intimal flap and drop it into the true lumen.
- Shaping the back of a stiff wire to J shape and using (Road runner, glidewire, confianza etc.)

- Using the proximal portion of a V18 wire formed after cutting the proximal loose part with a clamp forceps.

\section{Recommendations for atherectomy}

Excisional atherectomy is mostly used, and in this technique, our main goal is to prevent barotrauma and plaque shift that occur during balloon angioplasty. Treatment of severely calcified SFA lesions with atherectomy devices is still difficult and there is a risk of embolization. SilverHawk atherectomy catheter (Medtronic) is the best atherectomy device designed for the treatment of new and restenotic atherosclerotic lesions. This device is used with 0.014 inch wire. This device is used with 7-8 F sheath depending on the size. It has a blade rotation speed of $8000 / \mathrm{min}(10)$.

The patients who need to use a distal filter in chronic total occlusion of SFA are as follows [11]:

- Thrombotic load with sufficient landing area or any large plate graft stenosis, all bypass graft occlusion and thrombosis

- All cases of SFA stent occlusion, thrombosis, or SFA instent restenosis with adequate landing area

- Patients who experience acute worsening of their symptoms within 2 weeks, and these patients mostly have fresh thrombus.

- Most complex long SFA lesions with an adequate popliteal segment single vessel flow

- Lesions judged to be ulcerative

- Patients with critical leg ischemia with at least 3mm infrapopliteal artery

- Critical leg ischemia with an adequate popliteal segmented single vessel flow and severe proximal SFA disease.

It means enough landing area; it means reaching the disease-free area over 5-10 $\mathrm{mm}$ above the infrapopliteal trifurcation with a wire [11]

\section{New technologies in femoropopliteal lesions}

Intravascular lithoplasty system in peripheral artery disease: In this system, balloon angioplasty is performed with sound waves and calcified plaque is broken. With this system, calcified plaques, which are difficult to treat with drug-coated balloons or stents, are treated without damage to the vascular wall thanks to the low pressure balloon with the effect of sound waves. Shockwave peripheral intravascular system is a device known as M5, which contains over the wire balloon, 3.5-7 mm in diameter, $60 \mathrm{~mm}$ in length, can be used with a $6 \mathrm{~F}$ or $7 \mathrm{~F}$ sheat. Recently, 4 additional catheter sizes $(2.5 \mathrm{~mm} ; 3 \mathrm{~mm}, 3.5 \mathrm{~mm}$ and $4 \mathrm{~mm}$; $40 \mathrm{~mm}$ length, compatible with $5 \mathrm{~F}$ sheat, known as S4) have been defined. The S4 lithotripsy device has a smaller 
balloon catheter than the M5, has a lower profile, is hydrophilic coated, and has a longer shaft length. These device features are extremely important in treating contraleteral distal peripheral calcified lesions. There are 4-5 lithotriptors in the devices we mentioned. However, in the $\mathrm{S} 4$ device, 2 of the litrotriptors are in the middle. This property is very important in optimizing the intravascular lithotripsy effect on target calcified plates. In order to transmit the shock waves, the isotonic-contrast filled balloon is inflated at subnominal pressure $(4 \mathrm{~atm})$ in the calcified peripheral vein. Then, the shock waves are generated from the generator by pressing the intravascular lithotripsy button. This lithotripsy device is advanced through the calcified vein. An average of $160-300$ shock waves are generated per device $[12,13]$.

\section{Pantheris lumivascular atherectomy system}

It is a directional atherectomy system including optical coherence tomography. Provides three-dimensional visual guidance with optical coherence tomography. In addition, optical coherence tomography has the benefit of reducing damage to the vessel wall during plaque removal [14]. Pantheris Lumivascular Atherectomy system consists of catheter, sheat, lightbox imaging console and lightbox imaging sled. The catheter part is $135 \mathrm{~cm}$ and contains the optical fiber part of the optical coherence tomography $[14,15]$.

\section{Lithoplasty and pantheris studies}

35 patients with moderate to severe calcified femoropopliteal lesions were included in the DISRUPT PAD 1 study (2017). The mean initial stenosis of these patients was $76.3 \%$ and the mean lesion length was $61.5 \mathrm{~mm}$. $64.1 \%$ of these patients had severe calcification and the mean calcified lesion length was $80.3 \mathrm{~mm}$. The Lithoplasty system has been successful in all patients. The criterion for success was residual stenosis below $50 \%$. Residual stenosis averaged $23.4 \%$. 30-day patency rate was $100 \%$ while 6-month patency rate was $82.1 \%$ [16].

95 patients with moderate to severe femoropopliteal lesions (lesion length $15 \mathrm{~cm}$ and below) were enrolled in the DISRUPT PAD 1-2 study (2 phase study; 2016). Half of the patients had severe calcification. 94 of 95 patients underwent lithoplasty. Functional outcomes including walking improved significantly in 6 months. The average 6-month patency was reached in $91.6 \%$ of the patients. The average 6 -month patency rate was $76.7 \%$, while the recovery rate in 6 -month functional results was $96.8 \%$, regardless of the target vessel revascularization $[17,18]$.

158 patients with symptomatic superficial femoral artery lesions were included in the VISION (EvaluatIon of the Pantheris OptIcal Coherence Tomography Imaging Atherectomy System, 2017) study [14], which is associated with the Pantheris system. In this study, the mean lesion length was $58 \mathrm{~mm}$, the mean stenosis rate was $78.7 \%$, and the chronic total occlusion rate was $20.2 \%$. Cases with moderate to severe calcified lesions, patients with iliac artery lesions, cases with graft lesions, patients with restenotic lesions, cases with acute ischemia, and patients with thrombosed lesions were excluded from the study. In this study, the residual stenosis rate was below 50\% in 192 of 198 lesions with the Pantheris system. With the Pantheris system alone, the average initial stenosis rate fell from $78.7 \%$ to $30.3 \%$

\section{Conclusion}

Knowing the technical approach to FPL will contribute to the reduction of morbidity and mortality.

\section{References}

1. Endera M, Aboyans V, Bartelink ML, Baumgartner I, Clement D, et al. (2011) ESC Guidelines on the diagnosis and treatment of peripheral artery diseases: Document covering atherosclerotic disease of extracranial carotid and vertebral, mesenteric, renal, upper and lower extremity arteries: the Task Force on the Diagnosis and Treatment of Peripheral Artery Diseases of the European Society of Cardiology (ESC). Eur Heart J 32: 2851-2906. Link: http://bit.ly/3rcceH6

2. Karabay Ö, Karaçelik M, Yılık L, Tekin N, İriz AB, et al. (2012) Ischemic peripheral arterial disease: A screening survey. Turkish Journal of Thoracic and Cardiovascular Surgery 20: 450-457. Link: https://bit.ly/37ZwvZ9

3. Fowkes FG, Rudan D, Rudan I, Aboyans V, Denenberg JO, et al. (2013) Comparison of global estimates of prevalence and risk factors for peripheral artery disease in 2000 and 2010: a systematic review and analysis. Lancet 382: 1329-1340. Link: http://bit.ly/3kBem8Y

4. Sigvant B, Lundin F, Wahlberg E (2016) The risk of disease progression in peripheral arterial disease is higher than expected: a meta-analysis of mortality and disease progression in peripheral arterial disease. Eur $\mathrm{J}$ Vasc Endovasc Surg 51: 395-403. Link: http://bit.ly/3b9jFcN

5. Xu D, Zou L, Xing Y, Hou L, Wei Y, et al. (2013) Diagnostic value of anklebrachial index in peripheral arterial disease: a metaanalysis. Can J Cardiol 29: 492-498. Link: http://bit.ly/3r816ws

6. Aboyans V, Criqui MH, Abraham P, Allison MA, Creager MA, et al. (2012) Measurement and interpretation of the ankle-brachial index: a scientific statement from the American Heart Association. Circulation 126: 2890-2909. Link: http://bit.ly/3eclsi7

7. Tehan PE, Santos D, Chuter VH (2016) A systematic review of the sensitivity and specificity of the toe-brachial index for detecting peripheral artery disease Vasc Med 21: 382-389. Link: http://bit.ly/3kEVQg6

8. Fujihara M (2016) Angiography and Endovascular Therapy for Femoropopliteal Artery Disease. Link: http://bit.ly/3e0hnhW

9. Sheiban I, Moretti C, Omedé $P$, Sciuto F, Sciuto F, et al. (2007) The Retrograde Coronary Approach for Chronic Total Occlusions: Mid-Term Results and Technical Tips \& Tricks. J Interven Cardiol 20: 466-473. Link: http://bit.ly/3bRdMzR

10. Kansal A, Long CA, Patel MR, Jones WS (2019) Endovascular treatment of femoro-popliteal lesions. Clin Cardiol 42: 175-183. Link: http://bit.ly/3b72oRr

11. Allie DE, Hebert CJ, Patlola RR, Walker CM (2008) Embolic Protection Devices in Infrainguinal Interventions for Critical Limb Ischemia: A 24-Month Experience in 115 Patients. American Journal of Cardiology 601

12. Ali ZA, Nef H, Escaned J, Werner N, Banning AP, et al. (2019) Safety and Effectiveness of Coronary Intravascular Lithotripsy for Treatment of Severely Calcified Coronary Stenoses. Circ Cardiovasc Interv 12: e008434. Link: http://bit.ly/300iPIW

13. Hill JM, Kereiakes DJ, Shlofmitz RA, Klein AJ, Riley RF, et al. (2020) Intravascula Lithotripsy for Treatment of Severely Calcified Coronary Artery Disease. J Am Coll Cardiol 76: 2635-2646. Link: http://bit.ly/3q24ZQM 
14. Schwindt AG, Bennett JG, Crowder WH, Dohad S, Janzer SF, et al. (2017) Lower extremity revascularization using optical coherence tomographyguided directional atherectomy: final results of the EValuatlon of the PantheriS Optlcal COherence Tomography ImagiNg Atherectomy System for Use in the Peripheral Vasculature (VISION) Study. J Endovasc Ther 24: 355-366. Link: http://bit.ly/3sFAUIp

15. Ford $O$ (2017) Avinger snags CE mark for next-generation Pantheris, sets sights on ISR, BTK indications. BioWorld MedTech 21: 6. Link: http://bit.ly/300QthL
16. Brodmann M, Werner M, Brinton TJ, Illindala U, Lansky A, et al. (2017) Safety and performance of lithoplasty for treatment of calcified peripheral artery lesions. J Am Coll Cardiol 70: 908-910. Link: http://bit.ly/382Q5nb

17. 510(k) Premarket notification: summary [Internet]. Silver Spring (MD): U.S Food and Drug Administration; Sep 14, 2016. K161384: Shockwave medical lithoplasty system. Link: https://bit.ly/3020kUe

18. Safety and performance of the Lithoplasty ${ }^{\circledR}$ Technology System [Internet]. Fremont (CA): Shockwave Medical Inc; 2017. Link: http://bit.ly/3bOOLWg

\section{Discover a bigger Impact and Visibility of your article publication with}

\section{Peertechz Publications}

\section{Highlights}

* Signatory publisher of ORCID

* Signatory Publisher of DORA (San Francisco Declaration on Research Assessment)

* Articles archived in worlds' renowned service providers such as Portico, CNKI, AGRIS, TDNet, Base (Bielefeld University Library), CrossRef, Scilit, J-Gate etc.

* Journals indexed in ICMJE, SHERPA/ROMEO, Google Scholar etc.

- OAI-PMH (Open Archives Initiative Protocol for Metadata Harvesting)

* Dedicated Editorial Board for every journal

* Accurate and rapid peer-review process

* Increased citations of published articles through promotions

* Reduced timeline for article publication

Submit your articles and experience a new surge in publication services (https://www.peertechz.com/submission).

Peertechz journals wishes everlasting success in your every endeavours.

Copyright: @ 2021 Değirmenci H, et al. This is an open-access article distributed under the terms of the Creative Commons Attribution License, which permits unrestricted use, distribution, and reproduction in any medium, provided the original author and source are credited. 